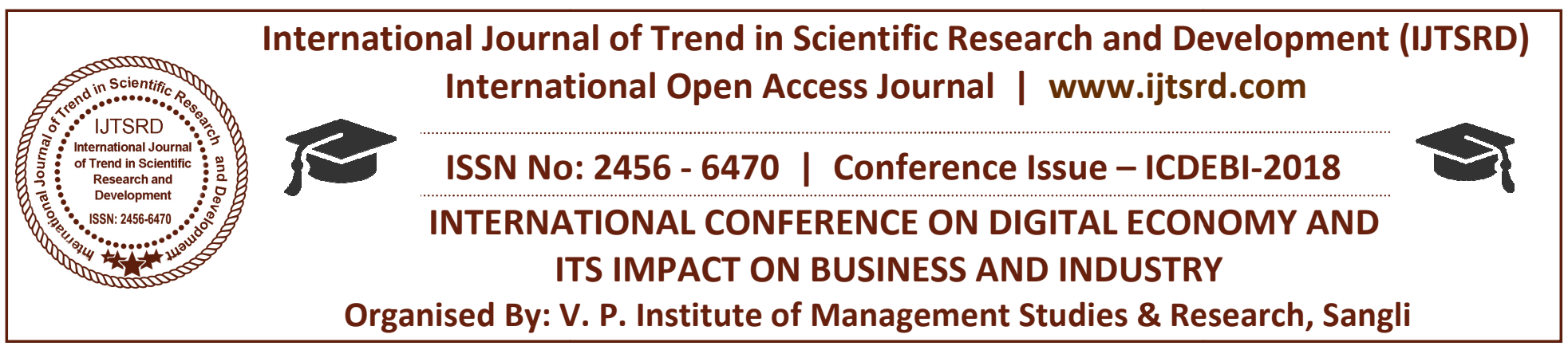

\title{
Artificial Intelligence \& its Role in Industry
}

\author{
Mrs. Jyoti M Bohra, Ms. Bhagyashri G Joshi \\ Assistant Professor, V. P. Institute of Management Studies \& Research, \\ Affiliated to Shivaji University, Sangli, Kolhapur, Maharashtra, India
}

\begin{abstract}
In today's digital era "Artificial intelligence" has been used in nearly all industries. AI has many applications in various different sectors like healthcare, aviation, IT, manufacturing etc. AI helps in reducing human errors, risks, best results with accuracy, does risky and/or hazardous jobs like bomb defusing, saves training cost. But at the same time AI has few risk factors like human intelligence can be replicated up to certain limit only, lesser jobs, hurt learners, affects human thinking powers.
\end{abstract}

\section{KEYWORD: Artificial Intelligence, Healthcare,} Manufacturing, IT.

\section{INTRODUCTION}

"Artificial Intelligence" as its name says its nonnatural intellect. If needs to be explained in simple words, it's just a work done by machines but by applying intelligence like humans do. As this intelligence is not natural like humans do have, we call it as artificial intelligence. This intelligence is added by humans in machines for accomplishing certain tasks. Through this intelligence machines can perform learning, planning, problem solving, perception, manipulation, cognitive abilities etc.

Broadly AI has two two major types,

1. Narrow AI.

2. General AI.

Narrow AI also known as Weak AI is focused on one narrow task. General AI also known as Strong AI or Full AI, where machines will have ability to perform general intelligent actions.

\section{Advantages:}

1. Chances of error are almost nil and greater precision and accuracy is achieved.

2. Space exploration

3. Fraud detection in smart card-based syst

4. Digital assistants

5. Diligence

6. Repetitive and time-consuming tasks efficiently.

7. Intelligent machines can be employed to do certain dangerous tasks. They can adjust their parameters such as their speed and time, and be made to act quickly, unaffected by factors that affect humans.

8. Sürgery simulators use machine intelligence in training medical professionals. AI can be used to simulate brain functioning, and thus prove useful in the diagnosis and treatment of neurological problems. As in case of any other field, repetitive or time-consuming tasks can be managed through the application of artificial intelligence.

9. Robotic radio surgery helps achieve precision in the radiation given to tumours, thus reducing the damage to surrounding tissues

\section{Drawbacks of AI:}

1. Over human intelligence and thinking power

2. unemployment

3. over human physical health

4. voice recognition issues

5. over young generation and children's mental and physical growth

6. machine/techno dependency

7. resources(like electricity)

8. machine will rule human and will not take any input from human even in most critical decisions 
9. risky in case of cyber attacks, when decisions are totally based on machines then in unpredictable crisis time its difficult or impossible to handle

10. while implementing AI human may miss out imp factors or possibilities to include in it

11. nuclear attacks through AI

12. cost incurred in the maintenance and repair

13. updated to suit the changing requirements

14. 14.ethics and moral values

15. data, but the storage, access, and retrieval is not as effective as in case of the human brain, do not get better with experience, like humans, Machines may not be as efficient as humans in altering their responses depending on the changing satiations',

16. Imagine robots working in hospitals. lack of emotions \& feelings

17. creative fields

18. Intuitive abilities that humans possess, the way humans can judge based on previous knowledge, the inherent abilities that they have, cannot be replicated by machines. Also, machines lack common sense.

19. Control of machines goes in the wrong hands, it may cause destruction. Machines won't think before acting. Thus, they may be programmed to do the wrong things, or for mass destruction.

20. fear of robots superseding humans, they might enslave us and start ruling the world

21. Greed to play God, man may destroy himself.

\section{b. Digital twins:}

A digital twin is a virtual model and useful when working with equipment from a remote distance. Sensors collect real-time data, working ailment or location. The system gets as well as processes data which is monitored by sensors.

\section{c. Computer vision:}

The most observant human assessor also may fail to find out faults which are of small or micro size. This is not the case with machine with camera. As its more accurate \& sensitive so it doesn't miss a bit of data also. After that the images will be sent to human to make decisions and improvements and after that sent back to machine.

\section{d. Case Study:}

Landing.ai, a start-up formed by Silicon Valley veteran Andrew $\mathrm{Ng}$, has developed machinevision tools to find microscopic defects in products such as circuit boards at resolutions well beyond human vision, using a machine-learning algorithm trained on remarkably small volumes of sample images. Once on line, the computer not only "sees," but also processes the information and learns from what it sees. If it spots a problem or defect, it sends an immediate alert, an AI process known as "automated issue identification. [1]

\section{e. Predictive maintenance:}

Predictive maintenance-as opposed to preventive maintenance-eliminates guesswork as the machines report their conditions on an up-to-theminute basis. It also saves businesses valuable time and resources, including labor costs, while guaranteeing optimal manufacturing performance. [1]

\section{Role of AI in IT industry: -}

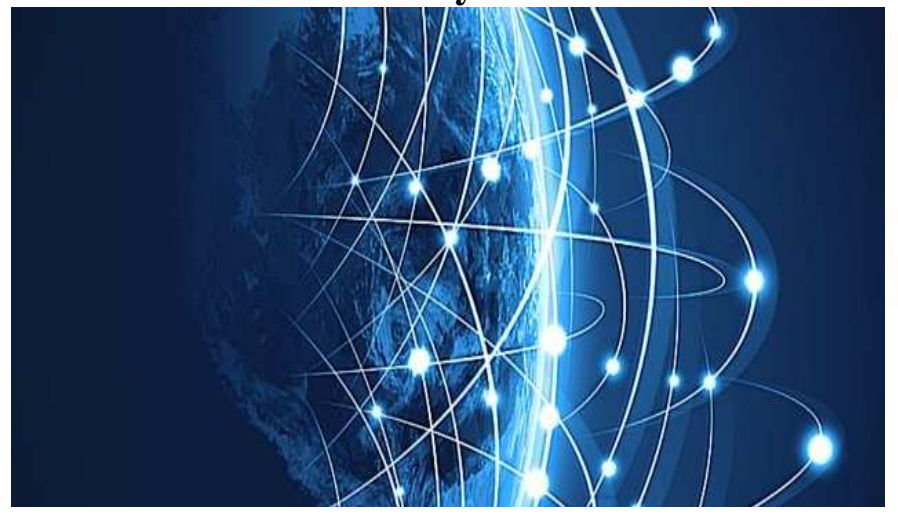

In manufacturing industry, it is majorly used for Realtime equipment maintenance and virtual product designs. Many types of software available in market which are specially designed for this sector.

\section{a. Generative design software:}

Are available for creating designs just by giving different parameters as an input. 
AI's major role is to make AI's teach other AI's and IT platforms without human intervention and/or efforts.

Artificial intelligence is being applied in almost every industry and its used up to maximum level in IT industry. In IT data collection, storage capacity and computational power is going on increasing. This massive data is valuable food for AI. This data can be analyzed, processed to comprehend new and changing trends.

Following are some examples of use of AI in IT industry:

1. Natural Language Processing (NLP):- The best example of NPL is speech recognition. It is process of converting phrases spoken by humans to machine readable format. Another example of Machine Vision is Text Translation.

2. Automation:- In this AI makes system work automatically. Such as repeatable and huge tasks which humans needs to perform can be automated. Example:- Robotic process automation.

3. Self-Driving Cars:- In this vehicle is auto piloted with the use of deep learning, computer vision \& image recognition.

4. Robotics:- Robots used to perform tasks which are difficult for humans. For example:- Robots are used in NASA for shifting huge objects in space

5. Machine Learning:- This is the process of automation of predictive analysis . Following are the 3 types,

$>$ Supervised learning,

$>$ Unsupervised learning \&

$>$ Reinforcement learning.

6. Machine Vision:- In this with the help of camera information can be captured as well as analyzed. It is used in range of applications.

7. In cyber security:- AI can identify any new and doubtful activities and/or weakness very fast and it can be then analyzed to find and stop/alleviate cyber-attacks.

\section{Role of AI in healthcare Industry: -}



AI has and will have a big role in healthcare industry. In this almost all traditional healthcare related tasks will be performed with the help of innovative data sciences.

Google Deep mind and G Suite aim to solve patient record complications by providing a secure, yet convenient way to access and transfer records while adhering to HIPAA rules and regulations. Deep mind will use block chain technology and cryptographythe same as crypto currencies - to ease the transferring and updating of patient medical records while maintaining security for healthcare professionals.

There are various applications which will collect patient records and processes them to perform diagnosis and help in giving ideal treatment suggestions as well as provide best personalized diet chart for patients. Applications will be even able to predict time duration required for curing illness.

Through predictive analysis, machine learning can look back at a patient's past medical records and find patterns that could suggest that the patient is headed toward a particular illness-like the previously mentioned cancer.

Doctors as well as patients will be benefited greatly with AI in healthcare sector. Doctors can utilize this information and treat patients for their faster recovery.

\section{Future of AI:}

With the help of huge data\& by applying statistics on that, one day AI will solve our world's biggest issue about climate change. We will have robots which can understand feelings of human, change its own sentiments, be friends with them and help them to stay happy.

\section{CONCLUSION:}

AI's is going on becoming smarter than human and after few decades it will be smartest. Which is indeed not a good thing. If $\mathrm{AI}$ is not dangerous right now but in near future it is definitely going to be dangerous. AI's will be the one who will take decisions for humans and also AI will control humans. Thus, AI and IT scientists must carefully as they create robots and computers.

Some worldwide standardized Rules, regulations and limitations should be set for use as well as in design 
International Journal of Trend in Scientific Research and Development (IJTSRD) ISSN: 2456-6470 | IF: 4.101

of AI for safety of humans. So that AI doesn't takeover and control humans in future.

\section{REFERENCES:}

1. https://www.forbes.com/sites/insightsintelai/2018/07/17/how-ai-builds-a-bettermanufacturing-process

2. https://www.techemergence.com/artificialintelligence-industry-an-overview-by-segment
3. https://www.zdnet.com/article/what-is-aieverything-you-need-to-know-about-artificialintelligence

4. https://becominghuman.ai/the-role-of-ai-inhealthcare-technology-6c33a6eee 18c

5. http://content.timesjobs.com/healthcare-sectordigital-job-profiles-to-trend-in2018/articleshow/61927270.cms

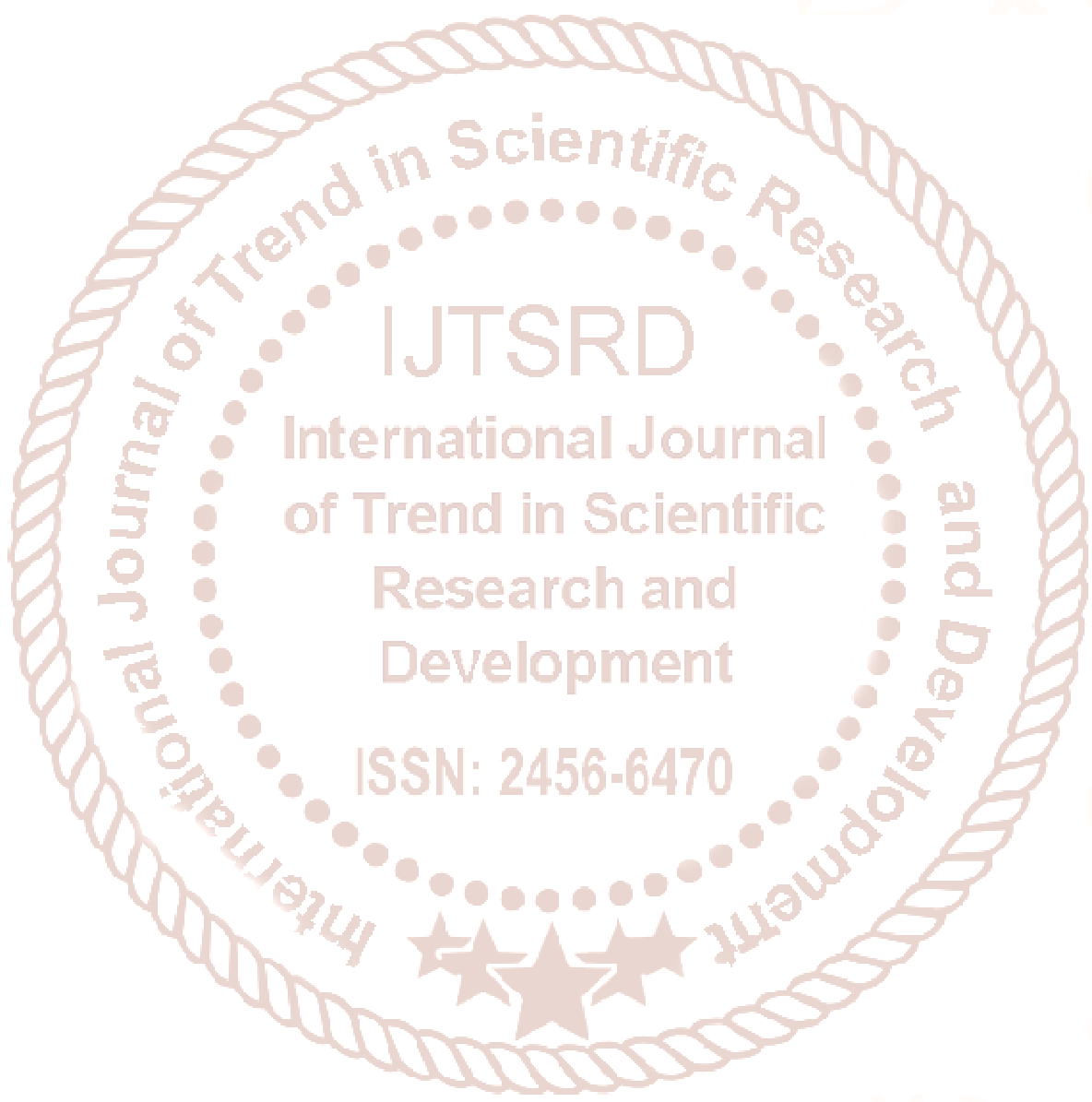

